\title{
Is the "Yoga For Kids" an Effective Way to Help Children with Autism Spectrum Disorder?
}

\begin{abstract}
Bohan Wan
Hefei No.1 High School, Hefei, Anhui, China

*Corresponding author. Email: guanghua.ren@gecacademy.cn

ABSTRACT

The study related to Autism Spectrum Disorder (ASD), Yoga for Kids program, and Cognitive Behavioral Therapy (CBT) among children with ASD from infancy(sixth month) to middle childhood (12 years old) (Domain). The research topic-- "is yoga for kids an effective way to help children with ASD-- is inspired by the real case of a 7 year-old child' s reaction to a 2-week yoga for kids program--he no longer avoids interaction with other adults and is more attached to his mother. Five articles are examined to prove that yoga for kids, which can be categorized as a part of Cognitive Behavioral Therapy (CBT), is an effective treatment for kids with ASD by enhancing their social engagement and preventing the future possibility that the children with ASD experience high levels of anxiety.
\end{abstract}

Keywords: Autism Spectrum Disorder, yoga, cognitive behavioral therapy (CBT), infancy, middle childhood, adults, males, females

\section{INTRODUCTION}

ASD refers to Autism Spectrum Disorder. Also, children with ASD always prefer to play alone and most of them have sleeping problems. What's more, the study has found a definition on APA that mentioned ASD is a complex developmental condition that involves persistent challenges in social interaction, speech, and nonverbal communication and restricted/repetitive behaviors (APA). In addition, it will mainly be reflected on children's ability to speak [1].

Yoga is a kind of effective sport that comes from Indian that helps cultivate muscular strength, endurance, and flexibility and enhances the practitioner's mental acuity and mindfulness [2]. Additionally, according to the website "Doylestown Health", yoga is useful in terms of concentration and self-esteem, which closely relates to two vital parts - emotion and ability of focus-functioning in the improvement of the speaking ability of children with an autism spectrum disorder. To be more specific, this study will focus on the therapy of three main impacts of ASD. The three parts are speaking ability, which refers to the capability of focus; the ability of social communication, which is related to emotion; and the last one is future social development.

Due to previous studies, ASD can be caused by traumatic childhood events [1]. At present, there are two main effective treatments for ASD. One is social therapy, such as yoga, music, and other methods to relieve symptoms by allowing children to communicate more with normal people. The other is biological therapy, like surgery or medication. As we mentioned before, children with ASD always have problems with sleeping, communication, social/parent-child engagement, and so on. What's more, they have high levels to experience anxiety and depression [3].

The scope of this study is broad. Although children between six months to twelve years old are more likely to have this mental disorder, ASD is a mental disease that includes anxiety and depression that everyone can experience. Therefore, the study's scope of people is adolescent, infants, middle childhood, adults, males, and females. In addition to the scope of races, this study mainly focuses on the therapy of ASD, especially yoga. And the study also mentioned Cognitive Behavioral Therapy(CBT) is effective to cure ASD. Thereby, yoga and CBT are also the scopes in this study.

Although these treatments can significantly reduce the symptoms like phobia, these methods can cause great discomfort for children with ASD [4]. Therefore, we are considering whether there is a way to effectively relieve these symptoms and keep these children feel comfortable during the treatment. And we finally find yoga, musical therapy, and CBT can be an effective 
tools for improving speaking ability and relieving possible anxiety for children with ASD.

\section{LITERATURE REVIEW}

\subsection{Causes}

To make the diagnosis of ASD as effective as possible, it is necessary to analyze both one's genes and experiences (nature vs. nurture) and connect them to his or her syndrome of ASD.

\subsubsection{Genes}

Gene is proved to be a strong possibility of causing ASD [5]. For instance, one of the researches done in Japan mentioned that the oxytocin receptor (OXTR) gene, which is secreted by the posterior pituitary gland and synthesized by the paraventricular and supraoptic nucleus of the hypothalamus, is a candidate gene of ASD. And their study supported that the OXTR gene plays a crucial role in the prevalence of ASD in Japan [6].

\subsubsection{Family}

Although there are lots of experiments and researches have done about ASD, there isn't a specific reason for the appearance of ASD. According to Mayo Clinic, Autism Spectrum Disorder might be caused by multiple factors - which are not easy to be noticed by parents and doctors - such as children's sex, family history (children in a family having a kid with ASD before), preterm babies, and even parents' age. For example, the divorce of parents, the relocation of family, and the unfair treatment and bullying in the school will all cause the child to suffer from ASD [7]. In other words, children who have experienced those unlucky things, they will be more likely to experience mental disease like anxiety and depression. Also, their ability of speaking and social communication will be limited, too. And these limitations will bring a significant difference to their future; For example, they cannot go to school because they cannot communicate with peers. Meanwhile, if a person had a mental illness before, such as depression and anxiety, then he or she is more likely to experience ASD [5]. Concretely, a trauma in childhood has a high probability of causing children to suffer from ASD.

\subsection{Social Impacts}

Autism Spectrum Disorder will not only cause children's speaking impairment and low social engagement as most people perceived, but also has further impacts on these children's future development. A recent study has shown that children with ASD had a high probability to experience comorbid psychological disorders, especially anxiety and externalizing disorders. And a study shows that such children's co-occurring psychological disorder is caused by children's impairment of speaking ability - pragmatic language deficit-which is exactly one of the main syndromes of the Autism Spectrum Disorder [8]. Additionally, social competence-usually is considered low social engagement - is well known as another syndrome of children with ASD, what people usually neglect is such problems on social competence make these children harder to socially integrate into the environment. And such impacts on these children vary in different groups of children with ASD. A study shows that children with ASD aside from language impairment and intellectual disability are more difficult to develop their social competence ability than children with ASD/alone do, which leads to significantly slower improvement in their future social development [9]. Moreover, children with ASD from different backgrounds might also have delayed social development due to their parent/family intervention. For instance, some Asian families might neglect their children's gaze avoidance-one syndrome of ASD-because they consider it as children's respect for adults by avoiding direct eye contact. In this case, children's ASD might not even be detected at an early age, making their social competence ability impossible to develop properly [10]. Both of the studies strongly suggest that early diagnosis and-especiallyearly/proper intervention to the children with ASD is necessary.

\subsection{Therapies—Creative Yoga Intervention}

\subsubsection{Improving Speaking Ability}

There is an effective tool--Creative Yoga Intervention--for the improvement of the speaking ability of children with Autism Spectrum Disorder (ASD). And a study conducted by Maninderjit Kaur supports this hypothesis that the movement-based, creative yoga intervention is effective for motor, social communication, and behavioral skills of children with ASD [5]. Gathering information from twelve children with ASD (from age 5 to 12) who accept 8 weeks of creative yoga intervention, the study tends to examine these children's changes in motor, imitation, and joint attention. Meanwhile, three of the changes accurately relate to specific practice in the creative yoga session: poses for practicing motor and imitation; social games for practicing imitation and communication skills; eye gaze training and breathing exercises for practicing joint attention. With the comparison of children's performance among early- mid-late- the period during the session, the result of the study indicates children's general improvement on the gross motor performance, imitation accuracy, and response to joint attention-which all attribute to the training during the yoga session. The study also shows children's train-specific 
improvement in communication skills which attributes to children's increasing joint attention. Regarding the previous study that attention functions in the improvement of the speaking ability of the children with Autism Spectrum Disorder, the result is supported by both concrete research information and professional theory [5]. Additionally, one of the comparison groups in the study, music intervention, suggests a greater positive and negative effect on children's social verbalization and interesting effect. Therefore, a welldesigned music intervention can be effective for treating children with ASD as well. Considering musical intervention as a part of creative yoga intervention as well, this study specifically proves that improvements in children with ASD are caused by multiple parts in the yoga session.

\subsubsection{Music In Yoga}

Music always plays an important role in yoga for kids. As illustrated above by the previous article-"Creative Yoga intervention for children with Autism Spectrum Disorder", the proper music intervention would facilitate the yoga for kids program, which more effectively leads to the improvement of social communication of children with Autism Spectrum Disorder (ASD). In addition, a study of home music environment provides another two benefits by the music intervention-- strengthen parent-child attachment; cause positive emotion of the children with ASD [7]. Conducting an online questionnaire including 340 families with children with typical (TD) and non-typical development, the study includes sufficient databases with children from the sixth month to 5 years old and accurate comparison groups among typical development (TD), Autism Spectrum Disorder (ASD), and Down's Syndrome(DS). According to data from the online survey, this study reports all families across the diagnostic groups (ASD, DS, etc.) have shared parentchild engagement in-home music that their children engage in various types of music activities [7]. Such parent-child music engagement then supports "parentchild affective attachment"--which is proved to be the key element for parent-involved treatment(more effective than child-only treatment) for children with ASD [11]. Additionally, how parent-child shared engagement supports parent-child attachment is by promoting the emotional connection between parents and their children--children and parents both experience positive emotion during this process thanks to the music activities. In the study "Factors affecting the ability to speak in children with autism spectrum disorders.", good emotion is another key element affecting the speaking ability of children with ASD. In this case, the point that music can benefit the social communication and speaking ability of children with ASD was reinforced again [5]. Conclusively, music is proved to have a positive effect on children with ASD as part of the creative yoga program.

\subsubsection{CBT And Yoga}

In addition to musical engagement, we have also found other ways to improve the condition of children with ASD. Cognitive Behavioral Therapy (CBT) is effective to relieve mental illnesses like depression and anxiety. What's more, people with ASD have a higher risk of experiencing these two symptoms according to this research paper. In this research, 32 participants who are diagnosed with General Anxiety Disorder (GAD) received treatment including CBT for 6 weeks. Finally, they found $\mathrm{CBT}$ can effectively reduce anxiety and elevate mood. Also, yoga can enhance CBT, which is Y-CBT will be more effective for people with ASD [2].

\subsubsection{The Improvement Of Mental Ability}

In addition, the article "Interventions based on the Theory of Mind cognitive model for autism spectrum disorder (ASD)" suggests the "Theory of Mind" (ToM) is the problematic part with autistic kids. To be more specific, autistic kids have a profound difficulty understanding the minds of other people, like their emotions, feelings, beliefs, and thoughts. We found articles that can give evidence that yoga can reduce these kinds of problems. For example, Doylestown Health illustrates that yoga can help autistic children with their focus and self-esteem. Also, Kaur said yoga can help children with ASD with their attention and verbalizing [5]. Therefore, the problem autistic kids have can be solved by yoga.

\section{LIMITATIONS AND FUTURE IMPLICATIONS}

\subsection{Limitations}

Three limitations are discussed in the research about Y-CBT [11]. Firstly, yet the study does not know how much change is attributable to yoga or how much CBT remains to be explored. Children with autism often have repetitive behavior, which can make them unable to complete different movements in a yoga session or even fear and resist therapy with many people. Whether they can understand yoga instructions is also a question. With traditional CBT, which focuses on the subjective removal of bad thoughts, yoga has been added to distract the brain from mental activity. However, people with ASD tend to be unusually focused on a subject of interest, which may counter the relaxing effects CBT is intended to achieve. Secondly, the physical movement aspect of yoga may account for higher dropout rates. Thirdly, the treatment only continued for 6 weeks, so maybe there is not enough time for yoga to work. CBT typically has between five and 20 sessions, depending 
on the direction of the treatment and the length of each session. Besides, normal people who focus on plasticity and breathing need 6-12 weeks of yoga practice to see the changes in their bodies, not to mention mental healing effect in children with autism; the duration of existing studies is generally 3-6 months, and there are not many long-term follow-up studies to ensure validity and continuity. There are also some limitations in research about ToM. Yoga rarely or never generalizes to novel contexts, and it is unclear whether there is the long-term maintenance of learned skills or developmental progression in learning.

\subsection{Future applications}

The study has two future suggestions for our research question. We can ask children with ASD to receive occupational therapy, which means working with kids to get through everyday tasks, like going to school or participating in class. In this way, they can be healthier and they will have less probability of getting further injury or disability. Also, it can be combined with school-based yoga programs, like the "Get Ready To Learn" program (GRTL). And it has shown significant decreases in teacher ratings of maladaptive behavior [6]. The other suggestion is that parents could play with children when they do yoga since Parents' engagement makes CBT more effective which cannot be achieved by child-only engagement. And activities such as the home music environment had also suggested future research for the relation between parent-child attachment and parent-child effective attachment across families with diagnosed children.

\section{CONCLUSIONS}

In ameliorating the problem of with ASD children's great speaking, sleeping issues, and alienation from society, the study focuses on treatments for ASD, specifically yoga, and cognitive behavioral therapy (CBT). It is discovered that through various poses and breathing and relaxing strategies, yoga is indeed useful for children with ASD in terms of their speaking ability, attention, and understanding the minds of other people by raising body awareness. Yet the study is still concerned regarding the ASD children's repetitive behavior, mind-distraction, elevating dropout rates while conducting yoga programs, and the short duration of the selected research, which may cast doubt on the validity and continuity of the result. The future application would be carried on by combining occupational therapy and school yoga programs. Besides, parents are suggested to participate in the yoga session with their children. This family music therapy environment also contributes to providing new and feasible directions for diagnosing children's family attachment and effective parent-child attachment.

\section{REFERENCES}

[1] Khalsa, M. K., Greiner, F. J. M., Hofmann, S. G., \& Khalsa, S. B. S. Yoga-Enhanced Cognitive Behavioural Therapy (Y-CBT) for Anxiety Management: A Pilot Study. Clinical Psychology \& Psychotherapy, 2015, 22(4), pp. 364 - 371. DOI: https://doi.org/10.1002/cpp.1902

[2] Saral, T. B., PhD. Yoga. Magill's Medical Guide (Online Edition), 2020

[3] Kerns, C., Newschaffer, C., \& Berkowitz, S. Traumatic Childhood Events and Autism Spectrum Disorder. Journal of Autism \& Developmental Disorders, 2015, 45(11), pp. 3475 - 3486. DOI: https://doi.org/10.1007/s10803-015-2392

[4] Ollendick, T., Muskett, A., Radtke, S. R., \& Smith, I. . Adaptation of One-Session Treatment for Specific Phobias for Children with Autism Spectrum Disorder Using a Non-concurrent Multiple Baseline Design: A Preliminary Investigation. Journal of Autism \& Developmental Disorders, 2021, 51(4), pp. 1015 1027. DOI: https://doi.org/10.1007/s10803-020$04582-5$

[5] Kałużna-Czaplińska, J., Żurawicz, E., \& JóźwikPruska, J. Focus on the Social Aspect of Autism. Journal of Autism \& Developmental Disorders, 2018, 48(5),pp. 1861-1867. DOI: https://doi.org/10.1007/s10803-017-3407-7

[6] Kaur, M. Creative yoga intervention for children with autism spectrum disorder (Order No. 10191810). Available from ProQuest Central; ProQuest Dissertations \& Theses Global. (1840889344). 2016

[7] Millau, M., Rivard, M., \& Mello, C. Immigrant Families, Perception of the Causes, First Manifestations, and Treatment of Autism Spectrum Disorder. Journal of Child \& Family Studies, 2018, 27(11), pp. 3468 - 3481. DOI: https://doi.org/10.1007/s10826-018-1180-7

[8] Rodas, N., Eisenhower, A., \& Blacher, J. Structural and Pragmatic Language in Children with ASD: Longitudinal Impact on Anxiety and Externalizing Behaviors. Journal of Autism \& Developmental Disorders, 2017, 47(11), pp. 3479 - 3488. DOI: https://doi.org/10.1007/s10803-017-3265-3

[9] Bennett, T. A., Szatmari, P., Georgiades, K., Hanna, S., Janus, M., Georgiades, S., . . . Thompson, A. Language impairment and early social competence in preschoolers with autism spectrum disorders: A comparison of DSM-5 profiles. Journal of Autism 
and Developmental Disorders, 2014 ,44(11), pp. 2797-808. DOI: http://dx.doi.org/10.1007/s10803014-2138-2

[10] Millau, M., Rivard, M., \& Mello, C. Immigrant families, perception of the causes, first manifestations, and treatment of autism spectrum disorder. Journal of Child and Family Studies, 2018, 27(11), 3468-3481.DOI: http://dx.doi.org/10.1007/s10826-018-1180-7

[11] Maemonah, S., Hamidah, Notobroto, H. B., Sulystiono, D., \& Widarti, L. Factors affecting the ability to speak in children with autism spectrum disorders. Journal of Public Health Research, 10(2), 178 - 181. 2021 DOI: https://doi.org/10.4081/jphr.2021.2236 